\title{
A COMPARATIVE STUDY ON THE PSYCHOSOCIAL AND TREATMENT FACTORS IN FREQUENCY OF EPISODES IN BIPOLAR AFFECTIVE DISORDER
}

\author{
Muhammad Issudeen ${ }^{1}$, P. G. Saji2
}

1 Junior Medical Consultant, Department of Psychiatry, Government Mental Health Centre, Kozhikode, Kerala. ${ }^{2}$ Additional Professor, Department of Psychiatry, Government Medical College, Kottayam, Kerala.

ABSTRACT
BACKGROUND
Bipolar disorder is a chronic psychiatric illness of an episodic and recurrent nature with marked mood and behavioural
dysfunction and causes substantial psychosocial morbidity, as it frequently affects independent living, vocational, and social
activities. But there is a relative dearth of Indian research about the factors associated with risk of recurrence in patients with
BPAD receiving treatment according to contemporary practice guidelines
The aim of this study was to assess the association of psychosocial and treatment factors with frequency of episodes in Bipolar
Affective Disorder (BPAD).

\section{MATERIALS AND METHODS}

A cross-sectional study consisted of first 120 subjects with bipolar disorder who availed psychiatry services in a general hospital setting in central Kerala from January 2014 to July 2014. Diagnosis was made by DCR-10 criteria. Data for 114 subjects with BPAD were analysed. Episode frequency was estimated as the number of episodes of depression, mania, hypomania and mixed per year of illness. Stressful life events were assessed by Presumptive Stressful Life Event scale and treatment adherence by Drug Attitude Inventory. Modified Camberwell Family Interview was used for assessing expressed emotions. Kuppuswamy's Socio Economic Scale was used for assessing socioeconomic status (SES).

\section{RESULTS}

Episode frequency was significantly associated with young age group, female sex, low educational status (Primary), unemployment, lower socioeconomic class, marital status (Single), number of children (Zero), earlier age at onset, family history of BPAD, high stressful life events, high expressed emotions, and poor treatment adherence. The association of comorbid general medical condition and psychiatric condition with episode frequency were not significant. The influence of religion, family type and comorbid substance use on episode frequency could not be commented upon.

\section{CONCLUSION}

Episode frequency was significantly associated psychosocial and treatment factors. Hence, to reduce the recurrence in BPAD, specific interventions are required to change the modifiable risk factors.

\section{KEYWORDS}

Bipolar Affective Disorder (BPAD), Recurrence, Episode frequency, Psycho-Social and Treatment Factors.

HOW TO CITE THIS ARTICLE: Issudeen M, Saji PG. A comparative study on the psychosocial and treatment factors in frequency of episodes in bipolar affective disorder. J. Evolution Med. Dent. Sci. 2018;7(09):1075-1081, DOI: 10.14260/jemds/2018/245

\section{BACKGROUND}

The illness severity of Bipolar Affective Disorder (BPAD) varies widely across affected individuals, ranging from relatively milder (fewer lifetime mood episodes with longer periods of inter-episode affective and functional recovery) to more severe (more chronic course with more frequent severe episodes and at the most, brief partial inter-episode improvement). 1

In BPAD, both biological (e.g., genes) and environmental (e.g., early life stress) factors may contribute to illness severity and chronicity.

'Financial or Other Competing Interest': None.

Submission 06-02-2018, Peer Review 18-02-2018,

Acceptance 20-02-2018, Published 26-02-2018.

Corresponding Author:

Dr. P. G. Saji,

Additional Professor,

Department of Psychiatry,

Government Medical College,

Kottayam, Kerala.

E-mail: drsajipg@gmail.com

DOI: $10.14260 /$ jemds $/ 2018 / 245$

(c) (i) $\ominus$
For instance, familial clustering aspects of bipolar illness course and early life stress has been associated with earlier age of onset, higher cycling frequency, suicidality, and greater prospective percent time ill in BPAD. ${ }^{2-4}$ Recent studies have reported that 19 to $76 \%$ of patients with BPAD suffer from persistent psychosocial impairment during the euthymic phase. ${ }^{5,6}$ Non-adherence to medication and early dropout from therapy also contribute to illness severity and chronicity. ${ }^{7}$

The onset of BPAD most commonly occurs in adolescence or early adulthood, which contributes to pervasive functional impairment and socioeconomic burden. Even treatment responsive patients, who remain well syndromally for extended periods of time, frequently demonstrate subthreshold symptoms and continuing psychosocial morbidity and cognitive impairment, suggesting that functional deterioration occurs and may be enduring. ${ }^{8}$

It is therefore no longer sufficient to focus treatment on the symptoms of acute episodes, but also to understand the course of the illness, reduce chronicity by preventing or delaying episode recurrence, and develop timely interventions by optimizing treatment strategies. ${ }^{9-11}$ 


\section{Aims and Objectives}

1. To study the association of episode frequency in BPAD with sociodemographic variables like age, gender, education, occupational status, socio economic status, religion, family type, marital status, number of children, and distance from home to hospital.

2. To study the association of episode frequency in BPAD with illness related variables like age at onset of BPAD, family history of BPAD, comorbid general medical condition, comorbid substance abuse, comorbid psychiatric conditions, stressful life events, expressed emotions from the care giver, and treatment adherence.

\section{MATERIALS AND METHODS}

This was a cross-sectional study conducted in the Department of Psychiatry, Government Medical College, Kottayam which is one among the premier multidisciplinary tertiary reference centres in Kerala. The study sample is constituted by first consecutive 120 patients admitted to psychiatry ward or attending OPD with Bipolar Affective Disorder for treatment and the study period was 6 months from $15^{\text {th }}$ January 2014 to $14^{\text {th }}$ July 2014.

\section{Inclusion Criteria}

1. Subjects diagnosed fulfilling the criteria for BPAD according to the Diagnostic Criteria for Research version of ICD-10 (DCR-10)

2. Subjects of age more than 18 years.

3. Subjects receiving treatment from Govt. Medical College Hospital, Kottayam, Psychiatry unit during the study period.

\section{Exclusion Criteria}

1. Subjects with substance induced mood disorder, mood disorder due to a general medical condition and schizoaffective disorder.

2. Subjects with recurrent depressive disorder, persistent mood disorders, other mood disorders and unspecified mood disorder.

3. Subjects or care givers not giving consent for the study.

\section{Study Tools}

Diagnostic Criteria for Research of ICD-10(DCR- 10): The Diagnostic Criteria for Research accompanying the ICD-10 (DCR-10) are designed for use in research; their content is derived from the Glossary to the chapter on Mental and Behavioural Disorders in the ICD-10 (Chapter V (F)).

\section{Kuppuswamy's Socioeconomic Status Scale 2012}

This scale takes account of education, occupation and income of the family to classify study groups in to lower, upper lower, middle, upper middle and upper socioeconomic status ${ }^{12}$.

\section{Presumptive Stressful Life Event Scale}

This scale ${ }^{13}$ consists of 51 life events that have been found to produce individual stress reactions. Each life event has got a numerical stress value which represent the degree of disruption that event causes in the average person's life. Accordingly, the total score obtained will be grouped into low stress (0-150), moderate stress (151-300) and high stress $(>300)$.
Treatment Adherent Scale (Drug Attitude Inventory)

Developed by AG Awad ${ }^{14}$, is a 30 -item inventory that focuses on the subjective effect of neuroleptic medications. It also addresses insight into illness.

\section{Scale for Measuring Expressed Emotions of the Care Giver}

It's a modified Camberwell family interview (CFI) ${ }^{15}$ which includes 30 items and which is again divided into positive and negative items and includes hostility, emotional over involvement and relative's critical comments with a score of 1-150. Accordingly, the expressed emotion is divided into high (66-150) and low (25-65).

\section{Specially Designed Proforma}

Specially designed proforma for collecting relevant socio demographic and psychiatric details

\section{Study Procedure}

All subjects were interviewed by the researcher and all the variables mentioned in the specially designed proforma completed using appropriate tools. Episode frequency calculated on the basis of total reported episodes of major depression, mania, mixed or hypomania per year of illness. ${ }^{16}$

\section{Statistical Analysis}

During statistical analysis six subjects from the total sample with illness duration of less than 2 years were excluded, because a small denominator may inflate the episode frequency. So the final sample constitutes only 114 subjects. Analysis was done in two phases, 1. Descriptive statistical analysis 2) inferential statistical analysis

After completing descriptive analysis, episode frequency was divided into four equal parts (quartiles-Q1, Q2, Q3, Q4) after arranging it an ascending order. So the subjects in the first quartile (Q1) have lowest frequency of episodes and subjects in the last quarter (Q4) have highest frequency of episodes. Then the psychosocial and treatment variables in lowest episode frequency group (Q1) and that of highest episode frequency group (Q4) were compared so as to find out the association between psycho social and treatment variables with episode frequency by inferential statistical analysis using appropriate statistical test given below.

Results on continuous measurements were presented on Mean \pm SD (Min-Max) and results on categorical measurements were presented in Number (\%). Significance is assessed at $5 \%$ level of significance. Analysis of variance (ANOVA) has been used to find the significance of study parameters between three or more groups of patients, Chisquare or Fisher Exact test has been used to find the significance of study parameters on categorical scale between two or more groups.

\section{RESULTS}

Episode frequency was distributed over the range of 0.21 to 2.14 episodes per year. The mean of episode frequency was 1.08 episodes per year with a SD of 0.51 episodes per year. In quartile distribution of episode frequency, $25.4 \%$ of the population belongs to low episode frequency group (Q1)$<0.67$ episodes per year) whereas $21.1 \%$ of total population belongs to high episode frequency 1.5 episodes per year. 


\begin{tabular}{|c|c|c|c|}
\hline $\begin{array}{c}\text { Episode } \\
\text { Frequency }\end{array}$ & $\begin{array}{c}\text { No. of } \\
\text { Subjects }\end{array}$ & $\%$ & Mean \pm SD \\
\hline$<0.67$ & 29 & 25.4 & \multirow{5}{*}{$1.08 \pm 0.51$} \\
\hline $0.67-1.00$ & 34 & 29.6 & \\
\hline $1.0-1.50$ & 27 & 23.7 & \\
\hline$>1.5$ & 24 & 21.1 & \\
\hline Total & 114 & 100.0 & \\
\hline
\end{tabular}

\section{Socio Demographic Variables and Episode Frequency in BPAD}

The age of the subjects in the sample ranged from 19 years to 63 years and most of the subjects in the study group were between 18 and 34 years of age (52.6\%) and least between 50 and 64 years of age. There is significant association between age and frequency of episodes in quartile distribution $(\mathrm{P}<0.001)$. It means that those having high episode frequency belong to young population (18-34 years of age). Among study participants $60 \%$ were males and $40 \%$ were females. In this study high episode frequency strongly was associated with female sex $(\mathrm{P}<0.001)$. Nearly half subjects $(49.1 \%)$ of the total sample had only primary school education and $32.5 \%$ achieved secondary (high school or plus two) education, while $14.9 \%$ finished graduation and only $2.6 \%$ had post-graduation. There is significant association between education and frequency of episodes. Subjects with education below graduation had high frequency of episodes (Q3 and Q4).

The study finds that majority of the subjects were employed (61.4\%) though below semi-professional level and unemployed were $33.3 \%$. Only $5.3 \%$ were self-employed like farming in their own land or running their own stationary stores. Unemployed subjects had significantly high episode frequency compared to employed subjects. Majority of the subjects (71\%) belongs to lower class and remaining $29 \%$ were from middle class. No single subject from upper class. Subjects belongs to upper lower class of Kuppuswamy's SES scale had significantly high episode frequency.

In terms of religion, $57.9 \%$ were Hindus, $39.5 \%$ were Christians and $2.6 \%$ were Muslims. Subjects belonged to Hindu religion had significantly high episode frequency compared to other religion. Except 6 subjects (5.3\%) who were from extended joint family, almost all subjects were from nuclear family. No single subject in the study group belongs to joint family (94.7\%). Subjects from nuclear family had significantly high episode frequency. Most of the subjects in the study group were single $(61.4 \%)$ followed by married (26.3\%) and divorced were $5.3 \%$ subjects of the total sample. Only $28.1 \%$ subjects of the study group had children because most of them were single. There is a significant association between marital status and episode frequency. Number of children negatively associated with frequency of episodes. In subjects belongs to high episode frequency had no children. The mean distance travelled by subject for receiving treatment is $26.57 \mathrm{~km}$ with a standard deviation of $10.08 \mathrm{~km}$. More than one third of the subjects (36\%) need to travel a long distance of 30 to $50 \mathrm{~km}$. Subjects belongs to low frequency group (Q1) travelled more than $40 \mathrm{~km}$ from home to hospitals and that high frequency group were $28 \mathrm{~km}$ only and the difference is found to be statically significant

Illness related variables and episode frequency in BPAD: This study found that $51.8 \%$ of the subjects first became ill between 11 to 19 years of age (Adolescence) and 37.7\% became ill in the young adult hood (20 to 40 years of age).
Subjects with adolescent age of onset had higher episode frequency.The mean of total number of episodes in lowest quartile(Q1) is 5 and that of highest quartile was 21. Almost half $(46 \%)$ of the study population had a family history of BPAD in first or second-degree relatives. Subjects with family history of bipolar illness are significantly high in high episode frequency group

Among the subjects with co-morbid General Medical Condition $42 \%$ had diabetes mellitus type II followed by hypothyroidism (36 \%). 18\% had Hypertension and 12\% had seizure disorder. About $18 \%$ of total sample had other chronic medical illness like chronic obstructive lung diseases, bronchial asthma, ischemic heart diseases and poly cystic ovarian disease. There is no significant association between episode frequency and medical co-morbidities $(\mathrm{P}=0.147)$. Among the subjects with substance use almost all had nicotine use. Along with nicotine majority of the subjects had alcohol use $(75.8 \%)$ and $19.3 \%$ of the subjects had cannabis use. Substance use includes either harmful use of the substance or substance dependence. Subjects with substance use are significantly high in low episode frequency group.

Only 12 subjects had psychiatric co-morbidities excluding substance use disorders as per DCR 10 criteria. Out of these 6 subjects were diagnosed as mild mental retardation, 4 subjects had co-morbid borderline personality disorder and remaining 2 subjects had associated social phobia. There is no significant difference between lowest and highest quartiles in psychiatric co-morbidities. High stressful life event experienced were only $26.3 \%$. Remaining subjects scored moderate $(30.7 \%)$ and low (43\%) in stressful life event scale. High score in Stressful life events scale was significantly high in high episode frequency group than low episode frequency group. Nearly half $(43 \%)$ of the subjects in the study population experienced high expressed emotions from the care givers. High expressed emotions are significantly more in highest frequency group compared to lowest frequency group.in this study only $32 \%$ had treatment adherence. Treatment adherence is significantly low in highest episode frequency group (12.5\%) compared to that of lowest episode frequency group (79\%)

\section{DISCUSSION}

Association between Study Variables and Episode Frequency in BPAD as follows-

\section{Age}

This study shows a significant association of age group with frequency of episodes in BPAD $(\mathrm{p}<0.001)$. The age group of subjects between 18 to 34 years were $79 \%$ in high frequency group (Q4) compared to that of low (Q1) frequency group (48\%). As per STEP BD which is a multi-centric cohort study for a period of 2 years with a sample size of 2000 subjects over the age of 15 years with a diagnosis of either BPAD I or BPAD II or BPAD not otherwise specified or cyclothymia or schizoaffective disorder as per DSM IV TR criteria found that age was not associated with risk of recurrence of $\operatorname{BPAD}(\mathrm{p}=>0.05) .{ }^{17}$ The discrepancy with our study could be due to the difference in study population (multicentre v/s hospital based), sample size, study design and duration, as cited above and difference in inclusion criteria (included all types BPAD and schizoaffective disorder as per DSM IV TR where as our study included subjects as per ICD 10 criteria 
for BPAD only). Moreover, our study used a formula for calculating episode frequency as number of episodes divided by duration of mental illness. ${ }^{16}$ Since age is a denominator function in episode frequency formula; as the age decreases, the episode frequency increases.

\section{Gender}

In our study, the episode frequency was significantly high in female gender ( $79 \%$ in high frequency group) compared to males $(21 \%$ in high frequency group) whereas in low frequency group consisted of $72 \%$ males and $23 \%$ females $(\mathrm{P}<0.001)$. The study by Kessing LV et al which was a hospital based case register review study to estimate the risk of recurrence in BPAD conducted in Denmark from 1971 to 1993 and consisted of a large sample of 20350 subjects with primary affective disorder had shown increased risk of recurrence for women in $\mathrm{BPAD}(\mathrm{P}<0.001) .{ }^{18}$ Our study also shows similar finding though it differs in sample size, study population and study designs from the above study. But most studies do not show significant gender differences in episode frequency of BPAD including earlier ECA and NCS studies. ${ }^{16}$

\section{Educational Status}

Our study found a significant association between educational level and episode frequency. Subjects with primary education (58\%) were more in high frequency group compared to low frequency group where subjects having primary education (24\%) and graduation $(21 \%)$ were almost equal. The studies by Schoyen HK et al which is a case control study with a sample size of 257 subjects diagnosed with BPAD as per DSM IV criteria conducted at a hospital in Norway also showed low education levels in rapid cycling BPAD and high frequency RDD subjects, which is consistent with our studies. ${ }^{19}$ But STEP BD as cited above showed no association with educational levels and risk of recurrence. ${ }^{17}$ The difference between studies could be due to the type of population in whom the study was conducted. Since our hospital was mainly catering to low income group, the sample we studied had a low educational achievement in general. The difference in study design and diagnostic criteria used for inclusion of subjects also might be contributed to the discrepancy between the studies.

\section{Occupational Status}

The frequency of episodes was significantly associated with employment status of the subjects. In the study $66 \%$ subjects of the total sample were unemployed in high frequency group compared to employed subjects (33\%) whereas in low frequency group $75 \%$ were employed and $24 \%$ subjects were unemployed. NCS-R study by Merikangas et al, which is a nationally representative survey of mental disorders among English-speaking household residents ages 18 years and older in the continental U.S ${ }^{20}$.In this study the BPAD subjects were selected by applying Composite International Diagnostic Interview (SCID). This study also found unemployed status associated with high episode frequency in BPAD. Though there is a difference in study setting, study design, sample size and diagnostic criteria used for inclusion of subjects, both studies show similar finding.

\section{Socio Economic Status}

Our study found a significant association between socio economic status and episode frequency. All the subjects $(100 \%)$ in the high frequency group were belonging to upper division of lower class in Kuppuswamy's Socio Economic scale compared to that in the low frequency group (52\%). Though there is a difference with our study, the observation that high frequency episodes in low SES is consistent with studies by Laeticia eid et al which is a cohort consists of 652 individuals with BPAD as per DSM IV from 2 centres (Hamilton and Ottawa) and was primarily done to find out the relation between lithium response and SES. ${ }^{21}$ This could be due to the fact that higher socio economic classes have a better access to treatment and more likely to seek treatment. But the STEP BD observed that socio economic status was not associated with relapse or recurrence. ${ }^{11}$

\section{Religion}

In high frequency group about $71 \%$ were Hindus and Christians were only $25 \%$. This may be due to the fact that majority of the subjects in the sample were Hindus because of which all subjects in low frequency group also belonged to Hindu religion (100\%).So the association between episode frequency and religion could not be commented though the association is statistically significant. To our knowledge there are no studies showing difference in prevalence as well as frequency among various religion. In a cross-sectional observation study of follow up data from large $(n=334)$ cohort study of patients receiving care for BPAD, Cruz M et al, found significant association between higher rates of prayer or meditation and precipitation of mixed state in BPAD $(\mathrm{P}=<0.05)$ as well as lower rates of prayer or meditation in euthymic subjects. ${ }^{22}$ But depression and mania were not associated with religious involvement.

\section{Family Type}

In this study all the subjects in both high frequency and low frequency group were belonging to nuclear family. This could be due to the fact that majority of the subjects in the total sample were from nuclear family. So, the association between family type and episode frequency could not be commented though the difference between nuclear and extended joint family were statically significant in both the groups. To our knowledge, there are no studies available to compare the association between family type and episode frequency. But it is evident that nuclear family has a less social support compared to joint family. The earlier studies found that lack of social support has a predictive value in BPAD recurrences. ${ }^{23}$

\section{Marital Status}

Our study shows a significant association with episode frequency. About $50 \%$ of the subjects in high frequency group were single and $20 \%$ were separated whereas in low frequency group 55\% subjects were single and $41 \%$ were married. This also consistent with previous studies which showed low episode frequency in married population (ECA study). But STEP BD found no significant association between episode frequency and marital status. ${ }^{11}$ 


\section{Number of Children}

In the study, majority of the (72\%) subjects had no children followed by $23 \%$ subjects with 1 to 2 children. This could be due to the fact that majority subjects in the sample were unmarried. But there is a significant association noted with episode frequency and number of children. In high frequency group all the subjects (100\%) were having no children compared to low frequency group where $55 \%$ subjects had no children and $45 \%$ subjects with 1 or 2 children. This could be also due to the same reason as cited above. To our knowledge there is no literature available to compare these facts

\section{Distance from home to Hospital}

The study also shows a significant association with distance travelled and episode frequency. The mean distance travelled by subjects in high frequency group was $28 \mathrm{~km}$ with an SD of $6.03 \mathrm{~km}$ whereas in low frequency group it is $41.90 \mathrm{~km}$ with an SD of $13.38 \mathrm{~km}$. This indicates an inverse relationship between episode frequency and distance travelled by the subjects. To our knowledge no previous studies are available to compare these findings. But there are studies showing urban-rural difference in BPAD. The studies by J Peen et al showed 39\% higher prevalence of mood disorder in subjects residing at urban areas. ${ }^{24}$ According to ECA and NCS studies there is no urban-rural difference in the prevalence of BPAD.

\section{Age at Onset of BPAD}

Our study found a strong association between age at onset and episode frequency. The subjects in high frequency group had an age of onset at 16.17 years with an SD of 2.5 years while that in low frequency were 28.97 years with an SD of 13.88 years. The studies by Fishfalen et al which is a case control study consisted of 625 subjects with diagnosis of BPAD as per DSM IV criteria found that highest episode frequencies were seen among subjects with onset between ages 15 and 18 years. ${ }^{16}$ Similarly, age at onset was significantly lower among those in the highest quartile of episode frequency. Though there is a difference in study design, study population and sample size between these studies and our study, both studies showing similar finding.

\section{Family History of BPAD}

The family history of BPAD is strongly correlated with episode frequency in our study. In high frequency group about $78 \%$ subjects had positive family history whereas in low frequency group it is only $11 \%$. This association is consistent with previous studies by Fishfalen et al which assessed the association between familiality and episode frequency and found episode frequency was significantly correlated among probands and their affected relatives (intraclass $\mathrm{r}=0.56, \mathrm{~F}=1.53, \mathrm{df}=96,321, \mathrm{p}<0.004$ ), suggesting that more than $30 \%$ of the variance in episode frequency was accounted by family membership ${ }^{16}$. Though there is a difference in study method and sample size, both studies show a significantly higher episode frequency in subjects with family history of BPAD.

\section{Co-Morbid General Medical Condition}

In our study, the episode frequency has no significant association with co-morbid GMC $(\mathrm{p}=0.141)$; but chi square test shows a positive association. This finding was partly consistent with studies by Fishfalen et al for thyroid disease in which they found no significant association between episode frequency and thyroid diseases. ${ }^{16}$ But the study done by P. V. Magalhaes et al revealed strong associations between variables related to illness chronicity and medical burden in bipolar disorder. 25

\section{Co-morbid Substance Use}

In our study only $21 \%$ subjects in the high frequency group had substance use while $72 \%$ subjects in low frequency group had co-morbid substance use. This shows a significant negative correlation between substance use and episode frequency. But previous studies showed a high risk of recurrence in BPAD subjects with co-morbid substance use. ${ }^{26}$ The discrepancy with our study could be due to the fact that the majority of the subjects in the high frequency group in our study sample were females (78\%) who were not using any form of substance while low frequency consist majority of males $(72 \%)$ who had one or more substance use either harmful use or in dependence level (as per DCR 10 criteria).

\section{Other Co-Morbid Psychiatric Condition}

In our study there is no significant association found between co-morbid psychiatric condition and episode frequency. A study by Schaffer et al found co morbid anxiety disorder was associated with increased morbidity and treatment resistance in BPAD and as per studies by Kora et al, presence of co-morbid psychiatric conditions is a predictor for longer episodes.27, 28 The discrepancy between our study and previous studies could be due to the low prevalence of psychiatric co-morbidities in total sample population as well as the difference in study population. Other characteristics such as low educational status and substance use that leads to under reporting of symptoms of other co morbid psychiatric conditions and thereby contributing to the discrepancies with previous studies.

\section{Stressful life Events}

In our study, it was found that high stressful life events were significantly associated with episode frequency. In high frequency group about $75 \%$ subjects had high stressful life event while that in low frequency group were nil. The study by Ernst et al which was a retrospective study consisted of 54 subjects with BPAD had high episode frequency. ${ }^{29}$ So our study is also consistent with finding that indicated a strong relationship between stressful live events and increased likelihood of episode recurrence like previous studies. ${ }^{29,30}$

\section{High Expressed Emotions}

Our study shows a significant association of high expressed emotions with episode frequency. In high frequency group about $67 \%$ subjects were experienced high expressed emotions from the carers whereas in low frequency group it is only about $21 \%(\mathrm{p}=0.006)$. The studies by Simoneau et al rated the expressed emotions levels of the relatives of bipolar I patients during a family interaction with the Camberwell Family Interview (CFI) and found that bipolar patients from high expressed emotions families had more manic symptoms $(\mathrm{d}=.64$ ) and a trend toward more depressive symptoms ( $\mathrm{d}$ $=.57$ ) than those from low expressed emotions families. ${ }^{31}$ In addition, high expressed emotions families were more likely than low expressed emotions families to show complex 
negative interaction sequences. So, our study is consistent with the studies by Simoneau et al 31

\section{Treatment Adherence}

Our study found a significant association between treatment adherence and episode frequency. Only $12.5 \%$ subjects had treatment adherence in high frequency group while that in low frequency group were $79 \%$. The finding was consistent with previous studies by Luis Gutiérrez-Rojas et al in which $78 \%$ of the subjects with poor adherence had high episode frequency compared to $57 \%$ subjects with good treatment adherence $^{32}$. The STOP EM which was cited above, also consistent with findings in our study. ${ }^{10}$

\section{CONCLUSION}

This study found a significant association between most of the socio-demographic variables and illness related variables. So further research is warranted to know the influence of each psychosocial and treatment factors on the episode frequency of BPAD.

\section{Implication}

The study was focusing on the influence of psychosocial and treatment factors in episode frequency of BPAD. There were significant similarities and differences when it was compared with other Indian and western studies. Since most of the psychosocial and treatment variable were significantly associated with episode frequency in BPAD, a case control study after matching the other confounding variables cited above in both the groups in a large sample size could be considered as active areas for studies in the future. Researches on the influence of specific variable after matching the other variables in the groups are also needed. More research is also warranted in order to know the influence of psychosocial and treatment factors on the episode frequency of BPAD as there is a dearth of Indian studies.

\section{REFERENCES}

[1] Suppes T, Dennehy EB, Gibbons EW. The longitudinal course of bipolar disorder. Journal of Clinical Psychiatry 2000;61(Suppl 9):23-30.

[2] Craddock N, Sklar P. Genetics of bipolar disorder: successful start to a long journey. Trends in Genetics 2009;25(2):99-105.

[3] Garno JL, Goldberg JF, Ramirez PM, et al. Impact of childhood abuse on the clinical course of bipolar disorder. British Journal of Psychiatry 2005;186(2):121-5.

[4] Leverich GS, McElroy SL, Suppes T, et al. Early physical and sexual abuse associated with an adverse course of bipolar illness. Biological Psychiatry 2002;51(4):28897.

[5] Marangell LB, Dennehy EB, Miyahara S, et al. The functional impact of subsyndromal depressive symptoms in bipolar disorder: data from STEP-BD. Journal of Affective Disorders 2009;114(1-3):58-67.

[6] Tohen M, Hennen J, Zarate CM, et al. Two-year syndromal and functional recovery in 219 cases of first episode major affective disorder with psychotic features. Am J Psychiatry 2000;157(2):220-8.
[7] Jackson WC. The importance of facilitating adherence in maintenance therapy for bipolar disorder. The Journal of Clinical Psychiatry 2008;69(1):e3.

[8] Coryell W, Scheftner W, Keller M, et al. The enduring psychosocial consequences of mania and depression. Am J Psychiatry 1993;150(5):720-7.

[9] Judd LL, Schettler PJ, Akiskal HS, et al. Residual symptom recovery from major affective episodes in bipolar disorders and rapid episode relapse/recurrence. Arch Gen Psychiatry 2008;65(4):386-94.

[10] Yatham LN, Kauer-Sant'Anna M, Bond DJ, et al. Course and outcome after the first manic episode in patients with bipolar disorder: prospective 12-month data from the systematic treatment optimization program for early mania project. Can J Psychiatry 2009;54(2):105-12.

[11] Altman S, Haeri S, Cohen LJ, et al. Predictors of relapse in bipolar disorder: a review. Journal of Psychiatric Practice 2006;12(5):269-82.

[12] Kumar N, Gupta N, Kishore J. Kuppuswamy's socioeconomic scale: updating income ranges for the year 2012. Indian Journal of Public health 2012;56(1):1034.

[13] Singh G, Kaur D, Kaur H. Presumptive stressful life event scale. Indian J Psychiat 1984;26(2):107-14.

[14] Awad AG. Subjective response to neuroleptics in schizophrenia. Schizophrenia Bulletin 1993;19(3):609-18.

[15] Vaughn CE, Leff JP. The influence of family and social factors on the course of psychiatric illness. A comparison of schizophrenia and depressed neurotic patients. British Journal of Psychiatry 1976;129:12537.

[16] Fisfalen ME, Schulze TG, De Paulo JR, et al. Familial variation in episode frequency in bipolar affective disorder. Am J Psychiatry 2005;162(7):1266-72.

[17] Perlis RH, Ostacher MJ, Patel JK, et al. Predictors of recurrence in bipolar disorder: primary outcomes from the Systematic Treatment Enhancement Program for Bipolar Disorder (STEP-BD). Am J Psychiatry 2006;163(2):217-24.

[18] Kessing LV, Hansen MG, Anderson PK. Course of illness in depressive and bipolar disorders. Naturalistic study, 1994-1999. British Journal of Psychiatry 2004;185(5):372-7.

[19] Schoeyen HK, Birkenaes AB, Vaaler AE, et al. Bipolar disorder patients have similar levels of education but lower socio-economic status than the general population. Journal of Affective Disorder 2011;129(13):68-74.

[20] Merikangas KR, Akiskal HS, Angst J, et al. Lifetime and 12-month prevalence of bipolar spectrum disorder in the National Comorbidity Survey replication. Archives of General Psychiatry 2007;64(5):543-52.

[21] Eid L, Heim K, Doucette S, et al. Bipolar disorder and socioeconomic status: what is the nature of this relationship? International Journal of Bipolar Disorders 2013;1:9. 
[22] Cruz M, Pincus HA, Welsh DE, et al. The relationship between religious involvement and clinical status of patients with bipolar disorder. Bipolar Disorder 2010;12(1):68-76.

[23] Rosenfarb IS, Miklowitz DJ, Goldstein MJ, et al. Family transactions and relapse in bipolar disorder. Family Process 2001;40(1):5-14.

[24] Peen J, Schoevers RA, Beekman AT, et al. The current status of urban-rural differences in psychiatric disorders. Acta Psychiatr Scand 2010;121(2):84-93.

[25] Magalhães PV, Kapczinski F, Nierenberg AA, et al. Illness burden and medical comorbidity in the Systematic Treatment Enhancement Program for Bipolar Disorder. Acta Psychiatr Scand 2012;125(4):303-8.

[26] Rakofsky JJ, Dunlop BW. Do alcohol use disorders destabilize the course of bipolar disorder? Journal of Affective Disorders 2013;145(1):1-10.

[27] Schaffer A, Cairney J, Cheung A, et al. Community survey of bipolar disorder in Canada: lifetime prevalence and illness characteristics. Can J Psychiatry 2006;51(1):9-16.
[28] Kora K, Saylan M, Akkaya C, et al. Predictive factors for time to remission and recurrence in patients treated for acute mania: Health Outcomes of Manic Episodes (Home) study. Prim Care Companion J Clin Psychiatry 2008;10(2):114-9.

[29] Ernst CL, Goldberg JF. Clinical features related to age at onset in bipolar disorder. J Affect Disord 2004;82(1):21-7.

[30] Malkoff-Schwartz S, Frank E, Anderson BP, et al. Social rhythm disruption and stressful life events in the onset of bipolar and unipolar episodes. Psychol Med 2000;30(5):1005-16.

[31] Simoneau TL, Miklowitz DJ, Saleem R. Expressed emotion and interactional patterns in the families of bipolar patients. Journal of Abnormal Psychology 1998;107(3):497-507.

[32] Gutiérrez-Rojas L, Jurado D, Martínez-Ortega JM, et al. Poor adherence to treatment associated with a high recurrence in a bipolar disorder outpatient sample. Journal of Affective Disorders 2010;127(1-3):77-83. 